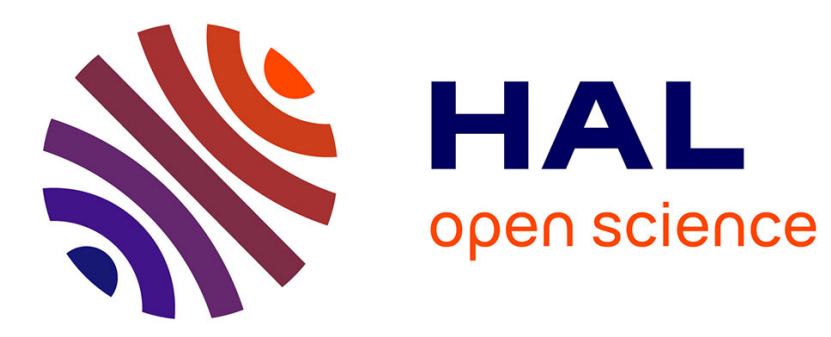

\title{
Interferometric Terahertz Wavefront Analysis
}

Emmanuel Abraham, Takayuki Ogawa, Mathilde Brossard, Takeshi Yasui

\section{To cite this version:}

Emmanuel Abraham, Takayuki Ogawa, Mathilde Brossard, Takeshi Yasui. Interferometric Terahertz Wavefront Analysis. IEEE Journal of Selected Topics in Quantum Electronics, 2017, 23 (4), pp.8400505 (1 - 5). 10.1109/JSTQE.2016.2638540 . hal-01495946

\section{HAL Id: hal-01495946 \\ https://hal.science/hal-01495946}

Submitted on 27 Mar 2017

HAL is a multi-disciplinary open access archive for the deposit and dissemination of scientific research documents, whether they are published or not. The documents may come from teaching and research institutions in France or abroad, or from public or private research centers.
L'archive ouverte pluridisciplinaire HAL, est destinée au dépôt et à la diffusion de documents scientifiques de niveau recherche, publiés ou non, émanant des établissements d'enseignement et de recherche français ou étrangers, des laboratoires publics ou privés.

\section{()(1)(2)}

Distributed under a Creative Commons Attribution - ShareAlikel 4.0 International 


\title{
Interferometric Terahertz Wavefront Analysis
}

\author{
Emmanuel Abraham, Takayuki Ogawa, Mathilde Brossard, and Takeshi Yasui
}

\begin{abstract}
Wavefront characterization of terahertz beams is useful for various applications such as terahertz spectroscopy and imaging. In this paper, we report on the aberration measurement of a terahertz beam issued from a quantum cascade laser. By using a terahertz camera and a two-wave noncommon path interferometer, we measured the wavefront distortions. As an example, we evaluated the Zernike coefficients giving the aberrations of spherical wavefronts induced by a converging lens. Associated with a deformable mirror, the sensor will open the route to terahertz adaptive optics.
\end{abstract}

Index Terms-Terahertz imaging, wavefront, optical aberrations, quantum cascade laser, interferometer.

\section{INTRODUCTION}

A WAVEFRONT sensor is an optical beam analyzer that determines the surface of equi-phase of an electromagnetic wave. For instance, the well-known Hartmann sensor was invented more than a century ago [1]. It makes it possible to locally measure the wavefront slopes of an incoming optical radiation by using a mask composed of a holes array placed just in front of a 2D sensor. At present, many other wavefront sensors are commercially available for visible and infrared light, based on intensity measurement or interference fringe analysis [2]-[4]. These systems are essential since they can provide the wavefront characterization for adaptive optics and a wide variety of applications including astronomy, ophthalmology and microscopy. These sensors are widely used to measure optical aberrations such as astigmatism, coma, spherical aberration, etc.

In the terahertz (THz) spectral domain, it is still challenging to fully measure the spatial profile and wavefront of a $\mathrm{THz}$ beam due to the lack of effective THz cameras. A few previous studies were oriented towards the measurement of $\mathrm{THz}$ wavefronts for both continuous wave and pulsed sources. In 2008, Bitzer et al. determined the beam profiles of $\mathrm{THz}$ pulses after passing through a hyperhemispherical silicon lens [5]. They observed an asymmetric spatio-temporal field dynamic in the focus of the lens attributed to a distortion of the incident $\mathrm{THz}$ wavefront.

This work was supported in part by the CNRS PICS Project no. 6507.

E. Abraham is with the Laboratoire Ondes et Matière d'Aquitaine, CNRS UMR 5798, Bordeaux University, Bordeaux Cedex 33000, France (e-mail: emmanuel.abraham@u-bordeaux.fr).

T. Ogawa and T. Yasui are with the Graduate School of Advanced Technology and Science, Tokushima University, Tokushima 770-8501, Japan (e-mail: ogawa@femto.me.tokushima-u.ac.jp; yasui.takeshi@ tokushima-u.ac.jp).

M. Brossard is with NeTHIS - New TeraHertz Imaging Systems, Mérignac 33700, France (e-mail: mathilde.brossard@ @ethis-thz.com).
However, they did not measure the $\mathrm{THz}$ wavefront but only the $\mathrm{THz}$ beam profile and their indirect and time-consuming method used a XY-scanning system in order to determine pointby-point the beam profile. Another aperture scanning device was proposed in 2013 to characterize the $\mathrm{THz}$ beam propagation in a time-domain spectrometer [6]. Associated with a Hartmann mask, it has been used to produce 2D topographical image of the THz wavefront. In 2012, Richter et al. proposed the first $\mathrm{THz}$ wavefront measurement with a Hartmann sensor and microbolometer camera [7]. The performance of the system was demonstrated by characterizing the wavefront of a $\mathrm{THz}$ beam emitted by a quantum cascade laser. Cui et al. analyzed the $\mathrm{THz}$ wavefront of a quantum cascade laser and a gas laser by using a Hartmann mask. However, they also used an indirect XY-scanning method to detect the radiation with a pyroelectric sensor [8], [9]. More recently, we reported on the wavefront measurement of $\mathrm{THz}$ pulses using a Hartmann sensor associated with a 2D electro-optic imaging system composed of a ZnTe crystal and a CMOS camera. We quantitatively determined the frequency-resolved deformations of planar and converging spherical wavefronts using the modal Zernike reconstruction least-squares method [10].

In this paper, we propose a simple interferometric method to measure the wavefront of a THz continuous wave. The measurement can be useful to control the optical alignment of a $\mathrm{THz}$ imaging system for which an incident planar $\mathrm{THz}$ wavefront is required in order to optimize, for instance, the focalization of the THz beam at the sample position. Basically, the system is based on a modified Mach Zehnder interferometer. It only requires a sensitive $\mathrm{THz}$ camera which is able to record the interferometric image generated by the superposition of two incoming $\mathrm{THz}$ waves. Then, using a simple fringe analysis, we can obtain the $\mathrm{THz}$ wavefront and its decomposition along the wellknown Zernike coefficients. As a demonstration, the method has been applied to measure the wavefronts and optical aberrations of convergent and divergent $\mathrm{THz}$ beams. Associated with deformable mirrors, the sensor will also open the route to $\mathrm{THz}$ adaptive optics.

\section{EXPERIMENTAL SETUP}

The experimental setup of the wavefront sensor is presented in Fig. 1. In our study, the source is a Quantum Cascade Laser (QCL, Longwave) providing a continuous wave at $3 \mathrm{THz}$ $(\lambda=100 \mu \mathrm{m})$ with an output power of $1.7 \mathrm{~mW}$. As many THz sources, the output beam is highly divergent and requires a specific beam shaping before any further utilization for $\mathrm{THz}$ imaging applications for instance. In our case, we used two 


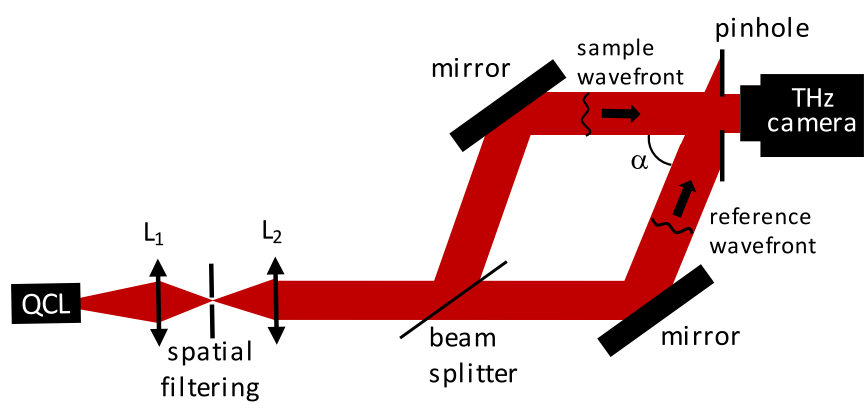

Fig. 1. General experimental setup for the analysis of planar THz wavefront $L 1$ and $L 2$ : plano-convex lens, focal distance of $50 \mathrm{~mm}$. Pinhole diameter $D=$ $3.2 \mathrm{~mm}$.

plano-convex lenses ( $L_{1}$ and $\left.L_{2}\right)$ and a spatial filtering to provide a planar beam.

The type of interferometer has been selected regarding the sensitivity of the THz camera. We used a NEC microbolometer array $(320 \times 240$ pixels, $23.5 \mu \mathrm{m}$ pixel size $)$ with a sensitivity of $1 \mathrm{nW}$. Therefore, to get a sufficient signal-to-noise ratio, it is better that the initial beam passes only once through a beamsplitter. For this reason, we selected a modified Mach Zehnder interferometer where the reference and sample beams travel along different paths before interfering. This shearing interferometer will be able to measure small aberrations since the fringe lateral shifting must be smaller to $2 \pi$ to avoid any phase ambiguity [11].

The interferometer is simply composed by a HFRZ-Si beamsplitter (reflection $=54 \%$, transmission $=46 \%$ for the vertical polarization) and two mirrors. The incoming $\mathrm{THz}$ beam is divided by the beamsplitter in order to form sample and reference beams. Both beams are recombined spatially in the plane of a pinhole whose diameter is set to $D=3.2 \mathrm{~mm}$ in the present study. The angle between the two interfering beams is noted $\alpha$. The intensity distribution in the plane of the pinhole is imaged with a magnification factor equal to one by the $\mathrm{THz}$ camera equipped with a $34 \mathrm{~mm}$ objective lens. In the present study, interference pattern is easily obtained owing to the long coherence length of the $\mathrm{THz}$ source (tens of centimeters).

\section{RESUlTS AND DISCUSSION}

\section{A. Optical Distortions Delivered by the Interferometer}

First, before characterizing the signal $\mathrm{THz}$ wavefront, we need to quantify the optical aberrations delivered by the interferometer itself, attributed to the beamsplitter and the two metallic mirrors.

Fig. 2(a) shows the interferogram corresponding to the experimental configuration presented in Fig. 1, with $\alpha=18^{\circ}$. Owing to the nearly equal amplitude of the electromagnetic waves and the sensitivity of the $\mathrm{THz}$ camera, the interference contrast is excellent (estimated to 85\%). First, we changed the value of the angle $\alpha$ in order to decide the best experimental configurations for the $\mathrm{THz}$ wavefront analysis. The idea is to obtain at least 10 fringes in the image. As shown in Fig. 3, the experimental fringe spacing $i$ is in excellent agreement with the theoretical relation
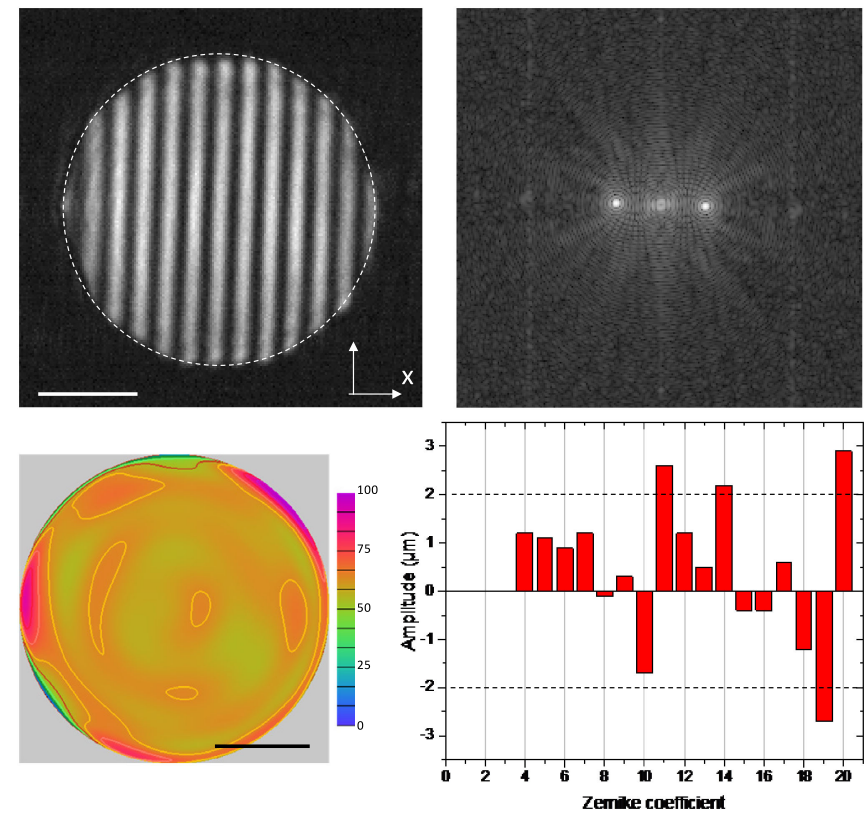

Fig. 2. Optical aberrations delivered by the interferometer. (a) Interferogram. White circle: image selection for FFT analysis. (b) Fourier domain image (c) Reconstructed wavefront. (d) Amplitude of the Zernike coefficients. Dotted black lines: limits of the measurement

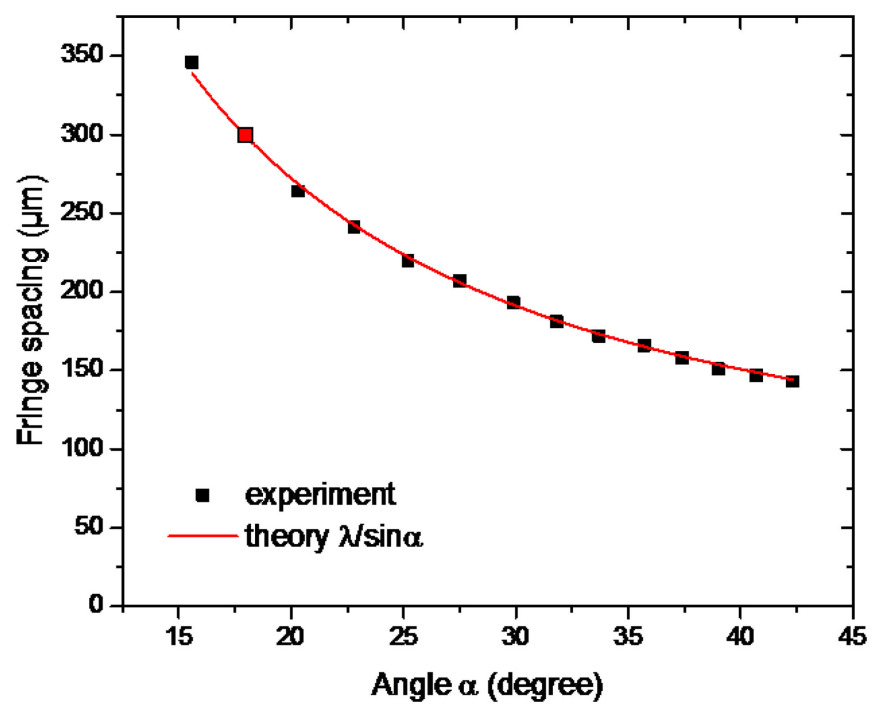

Fig. 3. Evolution of the fringe spacing as a function of the angle $\alpha$ between the two interfering waves. In the rest of our study, the angle $\alpha$ was fixed to $18^{\circ}$, indicated by the red square experimental point.

given by $i=\lambda / \sin \alpha$. In the rest of our study, the angle $\alpha$ was fixed to $18^{\circ}$, indicated by the red square experimental point in the figure.

Then, the analysis of the interference image is performed with the OpenFringe software as follows. First, we need to select the beam area in which the wavefront reconstruction will be calculated. In our case, this circular zone corresponds to the aperture of the pinhole ( $D=3.2 \mathrm{~mm}$ diameter), indicated by the white dotted circle in Fig. 2(a). Then, as shown in Fig. 2(b), 
TABLE I

ZERNIKE POLYNOMIALS

\begin{tabular}{llc}
\hline \hline $\mathrm{i}$ & \multicolumn{1}{c}{ Aberration } & $Z_{i}(\rho, \theta)$ \\
\hline 1 & Piston & 1 \\
2 & X tilt & $\rho \cos \theta$ \\
3 & Y tilt & $\rho \sin \theta$ \\
4 & Defocus & $2 \rho^{2}-1$ \\
5 & X astigmatism & $\rho^{2} \cos 2 \theta$ \\
6 & Y astigmatism & $\rho^{2} \sin 2 \theta$ \\
7 & X coma & $\left(3 \rho^{3}-2 \rho\right) \cos \theta$ \\
8 & Y coma & $\left(3 \rho^{3}-2 \rho\right) \sin \theta$ \\
9 & Spherical & $6 \rho^{4}-6 \rho^{2}+1$ \\
10 & X trefoil & $\rho^{3} \cos 3 \theta$ \\
11 & Y trefoil & $\rho^{3} \sin 3 \theta$ \\
12 & X 2nd astigmatism & $\left(4 \rho^{4}-3 \rho^{2}\right) \cos 2 \theta$ \\
13 & Y 2nd astigmatism & $\left(4 \rho^{4}-3 \rho^{2}\right) \sin 2 \theta$ \\
14 & X 2nd coma & $\left(10 \rho^{5}-12 \rho^{3}+3 \rho\right) \cos \theta$ \\
15 & Y 2nd coma & $\left(10 \rho^{5}-12 \rho^{3}+3 \rho\right) \sin \theta$ \\
16 & 2nd spherical & $20 \rho^{6}-30 \rho^{4}+12 \rho^{2}-1$ \\
17 & X tetrafoil & $\rho^{4} \cos 4 \theta$ \\
18 & Y tetrafoil & $\rho^{4} \sin 4 \theta$ \\
19 & X 2nd trefoil & $\left(5 \rho^{5}-4 \rho^{3}\right) \cos 3 \theta$ \\
20 & Y 2nd trefoil & $\left(5 \rho^{5}-4 \rho^{3}\right) \sin 3 \theta$ \\
\hline \hline & & \\
\hline
\end{tabular}

we computed the magnitude of the 2D Fast Fourier Transformation (FFT) of the interferogram. This is the usual procedure to perform a spatial frequency analysis of the interferogram. The output of this transformation represents the interference image in the Fourier or frequency domain where, each pixel represents a particular frequency contained in the spatial domain image. The magnitude of the Fourier domain image in Fig. 2(b) presents three horizontal bright spots since the original interferogram is composed of vertical fringes. The central spot can be ignored since it corresponds to the DC component of the Fourier domain image. The rest of the image, including the two remaining complex conjugated bright spots contain all the required information to calculate the phases shift between the sample and reference waves at the pinhole position.

The wavefront presented in Fig. 2(c) is almost planar even if some aberrations are visible in the edge of the beam, with a maximum wavefront distortion of about $100 \mu \mathrm{m}$ near the beam edge. This indicates that the optical elements of the interferometer, i.e. the $\mathrm{Si}$ beamsplitter and both mirrors, does not distort the wavefront of the incoming $\mathrm{THz}$ beam.

To quantify these aberrations delivered by the interferometer, we decomposed the wavefront $W$ into a linear combination of the Zernike polynomials [12]:

$$
W(\rho, \theta)=\sum_{i} a_{i} Z_{i}(\rho, \theta)
$$

where $(\rho, \theta)$ are the normalized polar coordinates $(\rho \leq 1)$ and the coefficient $a_{i}$ represents the amplitude of the aberration attributed to the polynomial $Z_{i}$, as referenced in Table I. Due to the non-collinear interaction between the reference and signal beams, the $\mathrm{X}$ tilt term is naturally very large $\left(a_{2}=575 \mu \mathrm{m}\right.$, calculated with a beam diameter of $D=3.2 \mathrm{~mm})$ ). For the wavefront reconstruction, the piston and XY tilt terms have been set to zero since they did not represent any intrinsic aberrations of the interferometer. Fig. 2(d) shows the amplitudes of the 20th

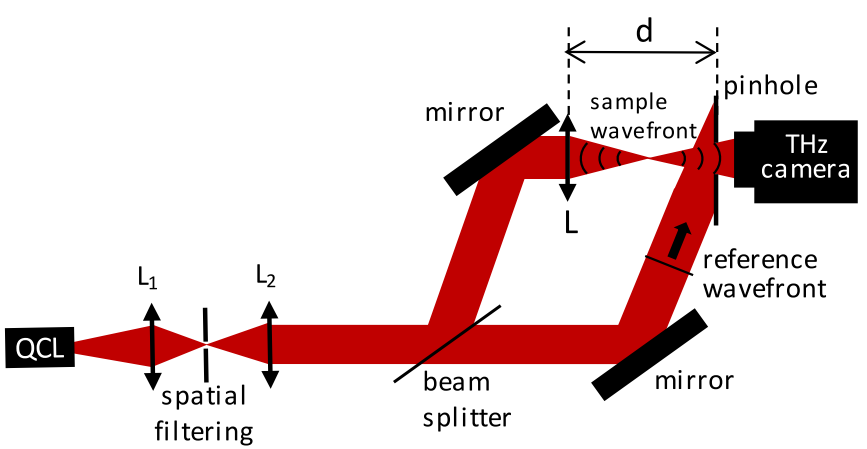

Fig. 4. Experimental setup for the analysis of spherical $\mathrm{THz}$ wavefront. $L 1$ and L2: lens, focal distance of $50 \mathrm{~mm}$. L: lens with $f^{\prime}=100 \mathrm{~mm}$. Pinhole diameter $D=3.2 \mathrm{~mm}$.

first Zernike coefficients, corresponding to the representation of the wavefront up to the second-order aberrations. All terms are smaller than $3 \mu \mathrm{m}$, i.e. $\lambda / 33$. The precision of our measurement has been estimated to $10 \%$, taking into account the accuracy of the fringe spacing determination (error of \pm 1 pixel corresponding to a fringe spacing of $(300 \pm 25) \mu \mathrm{m}$ for $\left.\alpha=18^{\circ}\right)$. We also estimated the minimum amplitude of the Zernike coefficients that the system is able to calculate. Considering a minimum tilt of $i=25 D=80 \mathrm{~mm}$ and the relation

$$
a_{2}=\frac{R}{\tan \left[\arcsin \left(\frac{\lambda}{i}\right)\right]}
$$

one gets $\mathrm{a}_{2}=2 \mu \mathrm{m}$ that can be generalized for any given Zernike coefficient. Therefore, we conclude that the minimum value of the Zernike coefficients that our system can measure is $2 \mu \mathrm{m}$, indicated by the two horizontal dotted black lines in Fig. 2(d). This shows the method makes it possible to reveal, at the limit of our detection limit, the following major intrinsic aberrations delivered by the interferometer: $a_{11}=2.6 \mu \mathrm{m}(\mathrm{Y}$ trefoil), $a_{14}=2.2 \mu \mathrm{m}(\mathrm{X} 2 \mathrm{nd}$ coma $), a_{19}=-2.7 \mu \mathrm{m}$ (X 2nd trefoil) and $a_{20}=2.9 \mu \mathrm{m}$ (Y 2nd trefoil). In the rest of this paper, these small aberrations will be subtracted in order to measure the signal $\mathrm{THz}$ wavefront without any perturbation coming from the interferometer itself.

\section{B. Wavefront Analysis of a $\mathrm{THz}$ Spherical Beam}

This section will present the wavefront analysis of THz spherical beams with different radius of curvature. The aim of the study consists in comparing the experimental defocus parameter to its theoretical prediction and the determination of all the other distortions.

The previous experimental setup has been modified by inserting, a plano-convex lens $\mathrm{L}$ with a focal distance $f^{\prime}=100 \mathrm{~mm}$ on the pathway of the signal beam (Fig. 4). By varying the distance $d$ between the lens and the pinhole it is possible to change the beam from converging $\left(d<f^{\prime}\right)$ to diverging $\left(d>f^{\prime}\right)$ wavefronts. Theoretically, assuming an incoming collimated Gaussian beam, the radius of curvature $R$ of the $\mathrm{THz}$ 


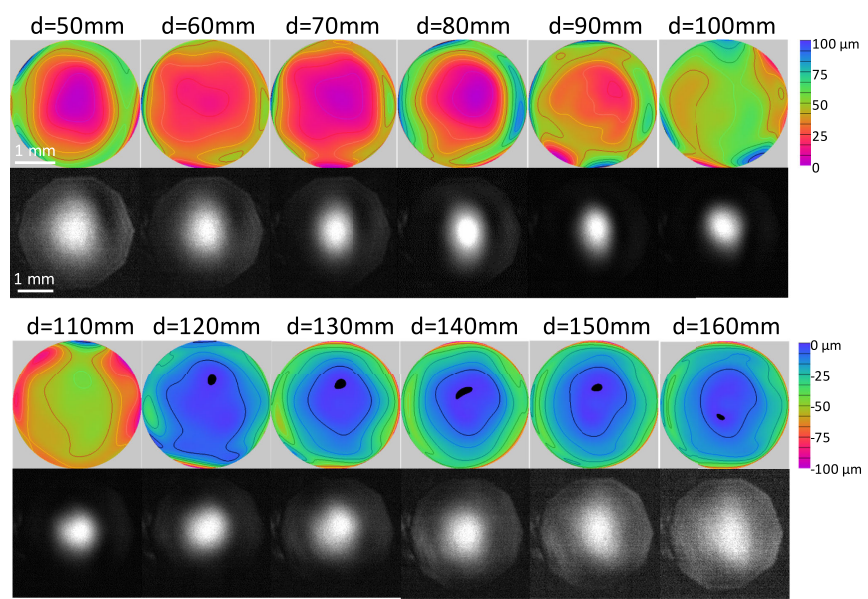

Fig. 5. THz wavefront (color) and THz beam (grey) as a function of the distance $d$ between the lens $L$ and the pinhole. The measurements are performed by the THz camera that images the pinhole plane. The distance $d=100 \mathrm{~mm}$ corresponds to the theoretical position of the beam waist.

wave at the pinhole position is given by

$$
R(d)=\left(d-f^{\prime}\right)\left[1+\left(\frac{z_{R}}{d-f^{\prime}}\right)^{2}\right]
$$

where $z_{R}=\pi w_{0}^{2} / \lambda$ is the Rayleigh length, with $w_{0}$ the radius of the beam waist. This shows that the beam waist is located at the pinhole position if $d=f^{\prime}$. Using the knife edge technique [13], we calculated $w_{0}=0.6 \mathrm{~mm}$, which gives $z_{R}=10.45 \mathrm{~mm}$. The interferogram is obtained by the interaction of this spherical sample beam and the reference beam. As already explained, the intrinsic optical aberrations of the interferometer will be removed.

Fig. 5 shows the evolution of the $\mathrm{THz}$ wavefront (color) as a function of the distance $d$ between the lens $L$ and the pinhole position, from $d=50 \mathrm{~mm}$ to $d=160 \mathrm{~mm}$. As expected, we can notice that the $\mathrm{THz}$ wavefront is convergent for $50 \mathrm{~mm}<d<$ $100 \mathrm{~mm}$ and then divergent for $100 \mathrm{~mm}<d<160 \mathrm{~mm}$. Around $d=100 \mathrm{~mm}$, the wavefront is nearly planar as it is also expected since this position corresponds to the theoretical beam waist of the optical setup. However, it is clear that this wavefront suffers from severe aberrations that will be discussed later. Fig. 5 also shows the beam transverse modes (grey color) as a function of the distance $d$. These images have been obtained by simply blocking the reference beam. Around $d=100 \mathrm{~mm}$, which corresponds to the focus point, we can observe the minimum beam diameter which has been measured at the $1 / \mathrm{e}^{2}$ maximum amplitude to $1.1 \mathrm{~mm}$.

First, we analyzed the evolution of the defocus as a function of the distance $d$. As previously explained, we decomposed the wavefronts into a linear combination of the Zernike polynomials (see Eq. (1)). The defocus coefficient $a_{4}$ is then related to the radius of curvature $R$ by the expression

$$
W(\rho)=2 a_{4} \rho^{2}=\frac{\left(\frac{D}{2} \rho\right)^{2}}{2 R}
$$

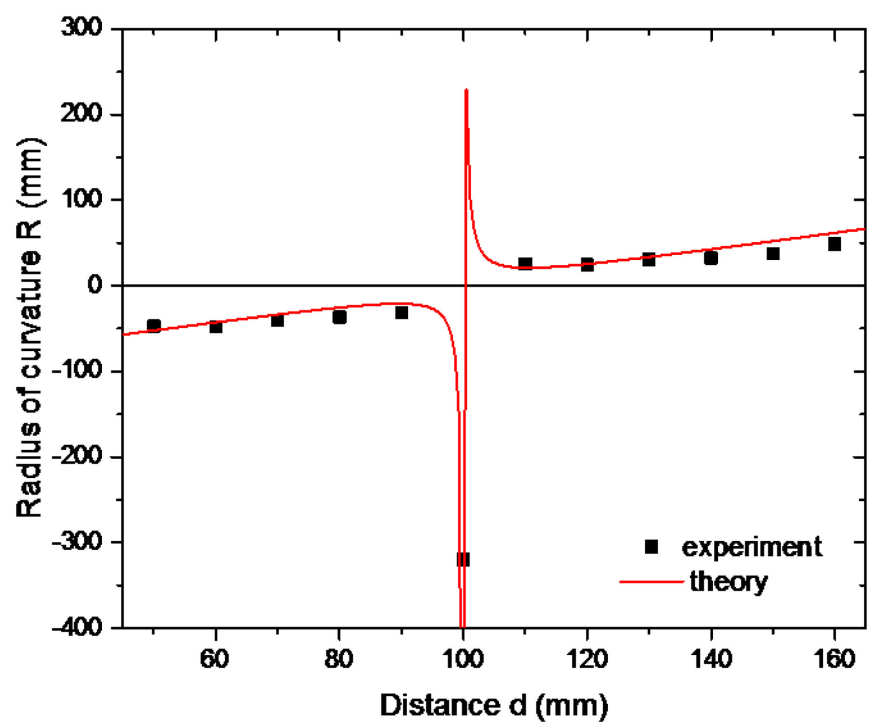

Fig. 6. Evolution of the amplitude of the radius of curvature $R$ as a function of the distance $d$ between the lens $L$ and the pinhole.

where $D$ is the diameter of the pinhole, taken as a reference for the beam diameter at the position where the wavefront is calculated. The Eq. (4) simply shows that it is possible to calculate the radius of curvature $R$ from the values of $D$ and $a_{4}$. The result is presented in Fig. 6 for different values of the distance $d$. It is in good agreement with theoretical values given by Eq. (3), which shows that $R(d)$ is proportional to the quantity $\left(d-f^{\prime}\right)$ except near the beam waist where the radius of curvature diverges since the wavefront becomes almost planar. For $d=50 \mathrm{~mm}$ for instance, the defocus coefficient is $a_{4}=(-13.6 \pm 2) \mathrm{mm}$, which correspond to $R=(-47 \pm 7) \mathrm{mm}$. This experiment indicates that our wavefront reconstruction is able to properly measure the beam defocus induced by the presence of the lens in the sample arm of the interferometer.

The final analysis concerns the ability of the interferometer to properly determine the other $\mathrm{THz}$ beam distortions as a function of the distance d. This measurement is particularly important for many applications since it is well-known that a distorted wavefront will not allow a strong focalization of the THz beam. Fig. 7 shows the amplitude of the Zernike coefficients $a_{5}$ (X astigmatism), $a_{6}$ ( $\mathrm{Y}$ astigmatism), $a_{7}$ (X coma), $a_{10}$ (X trefoil), $a_{11}$ (Y trefoil) and $a_{18}$ (Y tetrafoil) for different values of the distance $\mathrm{d}$ between the lens $\mathrm{L}$ and the pinhole. Out of the Rayleigh length $z_{R}=10.45 \mathrm{~mm}$, i.e. roughly $90 \mathrm{~mm}<$ $\mathrm{d}<110 \mathrm{~mm}$, we can notice that all the Zernike coefficients are around $5 \mu \mathrm{m}$, near the detection limit of our system that has been estimated to $2 \mu \mathrm{m}$. It means that out of the Rayleigh range, the convergent or divergent beam does not suffer from measureable aberrations. However, within the Rayleigh range, all the precedent Zernike coefficients increase up to $\pm 25 \mu \mathrm{m}$, indicating that near the beam waist the $\mathrm{THz}$ beam experiences severe distortions such as astigmatism, trefoil, coma and tetrafoil. This result is important since it opens the route to $\mathrm{THz}$ adaptive optics that has never been demonstrated yet. By using a deformable mirror on the $\mathrm{THz}$ signal beam pathway and by measuring the $\mathrm{THz}$ 


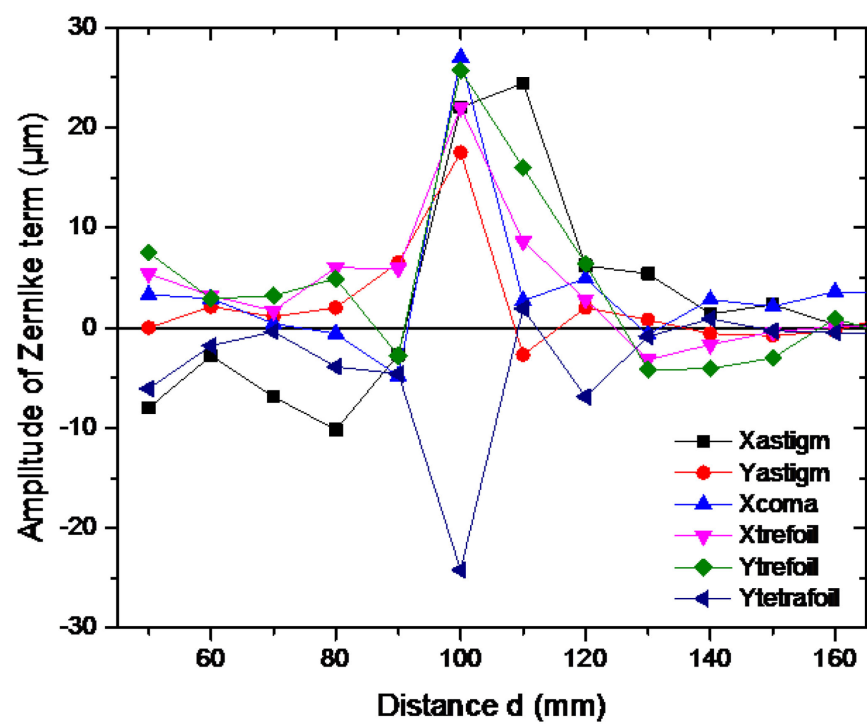

Fig. 7. Evolution of the amplitude of the Zernike coefficients as a function of the distance $d$ between the lens $L$ and the pinhole.

wavefront error after it has been corrected by the interferometer, we can form a closed loop wavefront sensor able to fully correct the beam distortion at the focus position, where a sample or a detector can be placed.

\section{CONCLUSION}

The paper concerns the characterization of $\mathrm{THz}$ wavefronts issued from a QCL source. With a modified Mach Zehnder interferometer, we determined the optical aberrations of the THz signal beam. Especially, important distortions have been measured near the focal spot of the $\mathrm{THz}$ beam, which can be problematic for future applications in spectroscopy or imaging for which a planar terahertz wavefront is required in order to optimize the focalization of the terahertz beam at the sample or detector position. Further, we believe that the interferometer could be associated with a deformable mirror and a realtime controller to open the route to $\mathrm{THz}$ adaptive optics.

\section{REFERENCES}

[1] J. Hartmann, "Bemerkungen über den Bau und die Justierung von Spektrographen," Z. Instrumentenkunde, vol. 20, pp. 47-58, 1900.

[2] J. M. Geary, Introduction to Wavefront Sensors. Bellingham, WA, USA: SPIE, 1995

[3] R. A. Gonsalves, "Phase retrieval and diversity in adaptive optics," Opt. Eng., vol. 21, pp. 829-832, 1982.

[4] J. Primot, "Three-wave lateral shearing interferometer," Appl. Opt., vol. 32, pp. 6242-6249, 1993.

[5] A. Bitzer, H. Helm, and M. Walther, "Beam-profiling and wavefrontsensing of THz pulses at the focus of a substrate-lens," IEEE J. Sel. Topics Quantum Electron., vol. 14, no. 2, pp. 476-481, Mar./Apr. 2008.

[6] J. F. Molloy, M. Naftaly, and R. A. Dudley, "Characterization of terahertz beam profile and propagation," IEEE J. Sel. Topics Quantum Electron., vol. 19, no. 1, Jan./Feb. 2013, Art. no. 8401508.

[7] H. Richter et al., "Terahertz wavefront measurement with a Hartmann sensor," Appl. Phys. Lett., vol. 101, 2012, Art. no. 031103.

[8] M. Cui, J. N. Hovenier, Y. Ren, A. Polo, and J. R. Gao, "Terahertz wavefronts measured using the Hartmann sensor principle," Opt. Express, vol. 20, pp. 14380-14391, 2012.
[9] M. Cui et al., "Beam and phase distributions of a terahertz quantum cascade wire laser," Appl. Phys. Lett., vol. 102, 2013, Art. no. 111113.

[10] E. Abraham, H. Cahyadi, M. Brossard, J. Degert, E. Freysz, and T. Yasui, "Development of a wavefront sensor for THz pulses," Opt. Express, vol. 24, pp. 5203-5211, 2016.

[11] D. C. Ghiglia and M. D. Pritt, Two-Dimensional Phase Unwrapping: Theory, Algorithms, and Software. Hoboken, NJ, USA: Wiley, 1998.

[12] W. H. Southwell, "Wave-front estimation from wave-front slope measurements," J. Opt. Soc. Amer, vol. 70, pp. 998-1006, 1980.

[13] A. E. Siegman, M. W. Sasnett, and T. F. Johnston Jr., "Choice of clip levels for beam width measurements using knife-edge techniques," IEEE J. Quantum Electron., vol. 27, no. 4, pp. 1098-1104, Apr. 1991.

Emmanuel Abraham was born in Reims, France, 1970. He received the Ph.D degree in physics from Bordeaux University, France, in 1997.

In 1997-1998, he joined the National Research Laboratory of Metrology, AIST, Tsukuba, Japan, as a Postdoctoral Research Fellow, and the FemtoSecond Technology project to study industrial applications of femtosecond lasers, including ultrafast optical Kerr gate. He has published more than 50 peer-reviewed journals, four patents, four book chapters, and more than 100 conference proceedings. From 1998 to 2013, he was an Assistant Professor in the Laboratoire Ondes et Matière d'Aquitaine, Bordeaux University, where he is currently a Professor. He was invited in Osaka University as an Invited Researcher in 2007 and the University of Tokushima as a Short-Term Invited Fellow Researcher of Japan Society for the Promotion of Science in 2010. His research interests include femtosecond time-resolved spectroscopy and nonlinear optics. He also has investigated the potential of terahertz $(\mathrm{THz})$ radiation for the analysis of objects related to cultural heritage such as paintings, sculptures, ceramics, etc., by using pulsed and continuous millimeter-wave sources for 2-D imaging and 3-D THz computed tomography. He developed intense THz sources and studied their applications for nonlinear spectroscopy. Thanks to the international collaboration with Tokushima University, he also developed innovative instrumentation for $\mathrm{THz}$ science and technology.

Takayuki Ogawa received the Bachelor's degree in mechanical engineering from Tokushima University, Japan, in 2015, where he is currently working toward the Master's degree in the Graduate School of Advanced Technology and Science. His areas of research are $\mathrm{THz}$ digital holography.

Mr. Ogawa is a member of the Optical Society of America and the Laser Society of Japan.

Mathilde Brossard was born in St Etienne, France, 1992. She received the Engineering degree from the Institut d'Optique Graduate School, France, in 2015. She started working toward the Ph.D. degree on $\mathrm{THz}$ wavefront measurement in Nethis Company in collaboration with Bordeaux University in 2016.

Takeshi Yasui received the Ph.D. degree in mechanical engineering from Tokushima University, Japan, in 1997.

From 1997 to 1999, he was a Postdoctoral Research Fellow in the National Research Laboratory of Metrology, Japan. He was with the Graduate School of Engineering Science, Osaka University, Japan, from 1999 to 2010, and was briefly with the University of Bordeaux I in 2007 and the University of Littoral Côte d'Opale in 2010 as an Invited Professor. He is currently a Professor in the Graduate School of Science and Technology, Tokushima University, and an Invited Professor in the Graduate School of Engineering Science, Osaka University. His research interests include $\mathrm{THz}$ instrumentation and metrology, nonlinear optical microscopy, and optical comb.

Dr. Yasui is a member of the Optical Society of America, the Japan Society of Applied Physics, the Optical Society of Japan, the Laser Society of Japan, the Japan Society of Medical Electronics and Biological Engineering, and the Japan Society of Mechanical Engineers. He received the Award for the Most Promising Young Scientist from the Optical Society of Japan in 1998, the Sakamoto Award from the Japan Society of Medical Electronics and Biological Engineering in 2006, the Optics Paper Award from the Japan Society of Applied Physics, and the Funai Award from the Japan Society of Mechanical Engineers in 2009 . 